\section{Encouraging surgical flexibility}

With implant cases, it is important to maintain surgical flexibility in order to offer the patient the best care possible.

With this in mind, the On 1 concept from Nobel Biocare is an effective choice for professionals looking to offer their patients an effective and versatile solution.

The On1 concept is designed to be used with any Nobel Biocare conical connection implant system, all of which have been created to give high primary stability and built-in platform shifting.

By moving the restorative platform from bone level to tissue level, the concept allows for easy attachment of restorative components and also makes impression taking far easier as the connection remains visible.

In addition, the On 1 concept's unique prosthetic connection ensures that only Nobel Biocare restorations can be used, giving patients peace of mind that their prosthesis will be supported by original components for the strongest solution.

For more information, call Nobel Biocare on 02087563300 or visit www.nobelbiocare.com/on1.

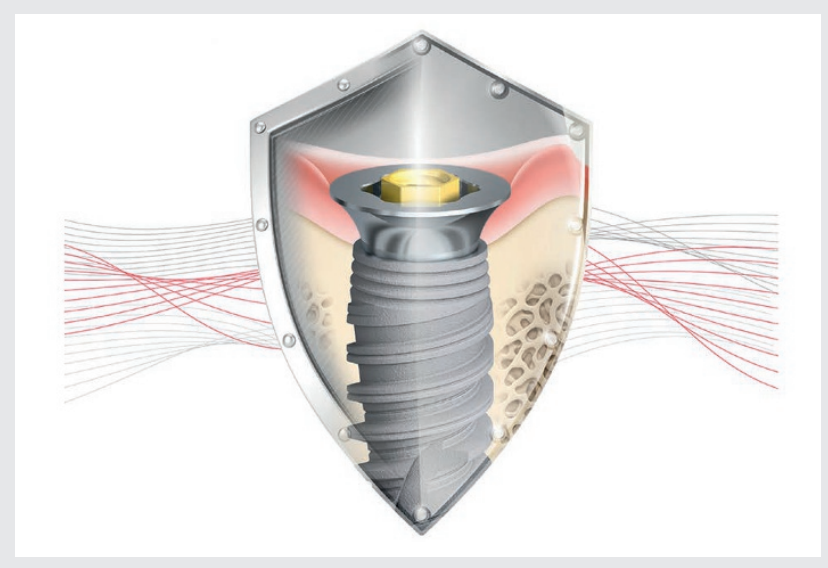

\section{Add-on innovation}

The W\&H Implantmed with the innovative new Osstell ISQ module enables clinicians to easily assess the stability of an implant in an instant.

This unique measuring system retrofits seamlessly and easily to the W\&H Implantmed surgical unit. It quickly evaluates and displays quantitative information including the Implant Stability Quotient (ISQ) and Resonance Frequency Analysis (RFA) to help clinicians to assess osseointegration.

Patients undertake a very simple and non-invasive procedure using a Smart Peg (which is inserted to the inside thread of the implant) and a measuring probe.

The Osstell ISQ module analyses the frequency at which resonance is reached using micro-vibrations and the readings provide clinicians with a safe, precise and reliable means of determining the optimum time to load an implant.

In addition, the W\&H Implantmed and Osstell ISQ module was an excellent communication tool, said the company. Clinicians no longer needed to be vague about the timing of implant loading because by using the measurements, clinicians could illustrate patient progress and explain more easily when more healing time was required.

More information is available by visiting www.wh.com/en_uk, by calling 01727874990 , or emailing office.uk@wh.com.

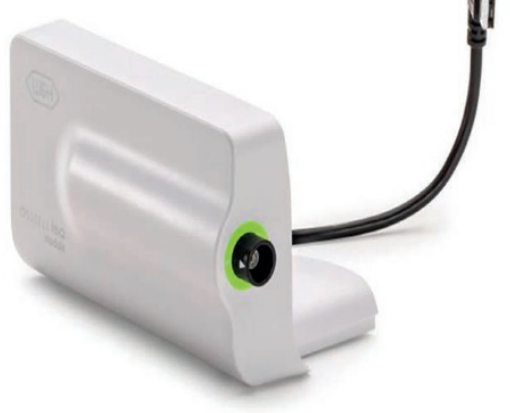

\title{
Homeless oral care conference
}

The Pathway Faculty Homeless and Inclusion Oral Health conference will be sharing insights into how to take action for inclusion for people experiencing homelessness and social exclusion at the BVSC Centre for Voluntary Action in Birmingham on 23 November 2018.

Did you know that $15 \%$ of people experiencing homelessness have attempted to extract their own teeth because of dental pain and that almost a third have accessed A\&E for their dental problems?

Have you ever wanted to use your dental skills to get involved with providing care for people experiencing homelessness or wanted to know how to set up your own homeless and inclusion oral health initiative?
These issues will be discussed at the November event at which keynote speakers will include Professor Andrew Hayward (UCL), Martin Burrows (Groundswell), Professor Ruth Freeman (University of Dundee) and Ben Atkins (Revive Dental Care) as well as experts with experience of homelessness and workshop speakers who will be sharing their 'how to guide' to inclusive oral health and dentistry. Join in to learn how you can get involved and take action to improve the oral health of people experiencing homelessness and social exclusion.

Tickets to the event can be found on the Pathway website: https://www.pathway.org. uk/training-and-events/ and for further information, email inclusivedentistrygroup@gmail.com.

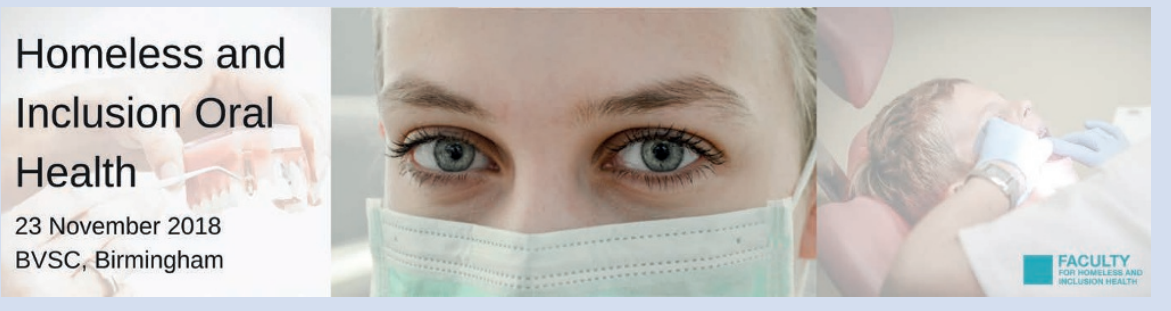

